\title{
Recent insights into the immunopathogenesis of psoriasis provide new therapeutic opportunities
}

\author{
Brian J. Nickoloff1 and Frank O. Nestle ${ }^{2}$
}

${ }^{1}$ Skin Disease Research Laboratory and Cardinal Bernardin Cancer Center, Loyola University of Chicago, Medical Center, Maywood, Illinois, USA. 2University of Zurich, Zurich, Switzerland.

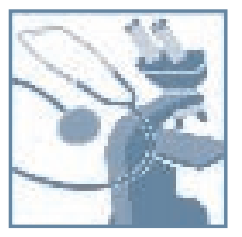

Chronic and excessive inflammation in skin and joints causes significant morbidity in psoriasis patients. As a prevalent $T$ lymphocyte-mediated disorder, psoriasis, as well as the side effects associated with its treatment, affects patients globally. In this review, recent progress is discussed in the areas of genetics, the immunological synapse, the untangling of the cytokine web and signaling pathways, xenotransplantation models, and the growing use of selectively targeted therapies. Since psoriasis is currently incurable, new management strategies are proposed to replace previous serendipitous approaches. Such strategic transition from serendipity to the use of novel selective agents aimed at defined targets in psoriatic lesions is moving rapidly from research benches to the bedsides of patients with this chronic and debilitating disease.

\section{Historical perspective, and clinical and histological features of psoriasis}

Psoriasis (OMIM 177900) is an ancient and universal inflammatory disease, initially described at the beginning of medicine in the Corpus Hippocraticum. Hippocrates (460-377 BCE) used the term psora, meaning "to itch." While the cause of psoriasis remains unknown, it appears to result from a combination of genetic and environmental factors. It is frequently inherited and passed from one generation to the next, but not following a classical autosomal mendelian profile. While it may have originally been confused with leprosy (lepra, "to scale"), it is generally easy to recognize psoriasis when it presents in one of three typical presentations: guttate, pustular, and plaque-stage. Figure 1 provides a clinical view of untreated chronic stationary plaques distributed on the lower back.

Approximately $2-3 \%$ of the population worldwide is afflicted by psoriasis. Several authors, including John Updike and Vladimir Nabokov, as well as British experimental dramatist Dennis Potter (The singing detective), carefully recorded the suffering that psoriasis, and side effects of its treatment, had on their lives. Not only can skin lesions be pruritic and disfiguring; in 10-30\% of patients there can also be nail dystrophy accompanied by psoriatic arthritis. The inflammation in the joints is similar in some ways to rheumatoid arthritis (RA), although in psoriasis it is a seronegative arthritis (no rheumatoid factor is present in the blood). Thus, the various clinical manifestations of psoriasis make it more than a dermatological nuisance, as it interferes

Nonstandard abbreviations used: Food and Drug Administration (FDA); highmobility group B1 (HMGB1); NK type T cell (NKT cell); programmed cell death 1 (gene) (PDCD1); regulatory single-nucleotide polymorphism (rSNP); rheumatoid arthritis (RA); receptor for advanced-glycation end products (RAGE); runt-related transcription factor (RUNX); single-nucleotide polymorphism (SNP); T cell receptor (TCR); very late antigen (VLA).

Conflict of interest: The authors have declared that no conflict of interest exists.

Citation for this article: J. Clin. Invest. 113:1664-1675 (2004).

doi:10.1172/JCI200422147.

The Science in medicine series is supported in part by a generous grant from the Doris Duke Charitable Foundation. with many normal daily activities, such as use of hands, walking, sleeping, and sexual activity. At least $30 \%$ of patients contemplate suicide, which places psoriasis on par with other major medical diseases such as depression, heart disease, and diabetes (1). The most frequent extracutaneous medical problem associated with psoriasis (besides arthritis of small joints) is the inflammatory bowel disorder Crohn disease (2).

Psoriasis can begin at any age, although epidemiological studies demonstrate that it most commonly appears for the first time between the ages of 15 and 25 years (3). It is a lifelong inflammatory disease with spontaneous remissions and exacerbations. In the plaque stage, lesions are characterized as being symmetrically distributed, and well demarcated from adjacent symptomless skin, with erythema topped by white-silvery scale (Figure 1). Many triggering factors initiate or exacerbate psoriasis, including bacterial pharyngitis, stress, HIV-1, and various medications (e.g., lithium and $\beta$-blockers). Microscopically, plaque-stage lesions reveal significantly thickened skin with confluent parakeratotic scale (Figure 1; insets compare symptomless skin with psoriatic plaques), loss of the granular cell layer, and increased number of epidermal cell layers with mitotic figures in basal-layer keratinocytes. Rete ridges, representing downward extensions of epidermis, are elongated, and the papillary dermal blood vessels appear tortuous and dilated. The inflammatory cell infiltrate may contain neutrophil collections in the epidermis, but a more consistent finding is the presence of $\mathrm{T}$ cells in the dermis and epidermis accompanied by increased numbers of dermal DCs, macrophages, and mast cells.

The history of the treatment of psoriasis is also of interest, as it reflects not only the uncertainty regarding its pathogenesis, but also the limited options available to clinicians in the past. Translational arcs of discovery for therapeutic agents used in psoriasis in the past, present, and future are displayed in Figure 2. Note that in the past, physicians used many different compounds and serendipitously found several that helped treat psoriasis, including arsenic (Fowler's solution) and ammoniated mercury. In many instances, neither the specific target nor the mechanism of action for the treatment was known. However, current treatments include a new wave of selective therapeutic 


\section{science in medicine}

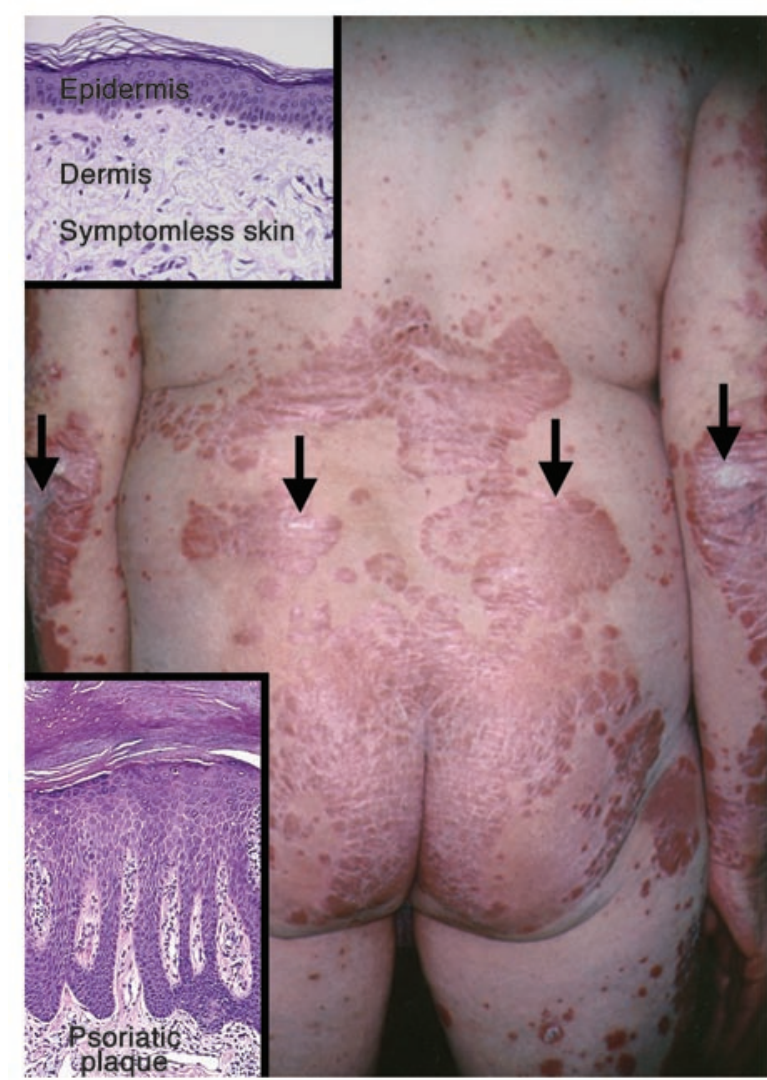

agents that emerged following introduction of cyclosporin A into dermatology clinics, reflecting our ever increasing understanding of psoriasis pathophysiology (4). The next section presents a brief overview of psoriasis pathophysiology, followed by detailed analyses of genetic, immunological, and animal-model developments; we then delve into new therapeutic advances. It should be noted up front that the current listing of psoriasis with other putative autoimmune diseases such as multiple sclerosis is largely based on essentially negative results, reflecting the failure of investigators to consistently isolate a specific infectious agent or identify circulating autoantibodies. As will become clear in the following sections, psoriasis challenges investigators to understand the interplay between genetic-susceptibility alleles and immunological and environmental factors that contribute to the chronic inflammatory process.

\section{Overview: pathophysiology of psoriasis}

As mentioned earlier, the cause of psoriasis remains unknown, and there is no cure. Several hypotheses have been advanced and models proposed over the years concerning its pathogenesis. In this section, a brief overview of psoriasis pathophysiology is presented. As one considers hypotheses for psoriasis, it is important to recognize at least three unique and characteristic features of this enigmatic disease: (a) Psoriatic plaques represent highly localized sites of dysregulated growth and inflammation, yet these sites almost never develop into, or harbor, malignant clones of keratinocytes, melanocytes, or T cells (5); (b) Despite significantly altered barrier function due to aberrant epidermal cell differentiation, psoriatic plaques are highly resistant to bacterial, viral, and fungal infections (6); (c) Psoriatic plaques, either

\section{Figure 1}

Clinical and histological appearance of stable chronic psoriatic plaques. Note the well-demarcated erythematous plaques covered by white-silvery scale (arrows), widely distributed on the lower back and extremities. Prepsoriatic symptomless skin is clearly demarcated from lesional skin. Psoriasis often develops at skin sites where minor trauma may occur, such as elbows and buttocks. Induction of psoriatic lesions by trauma is referred to as the Köbner phenomenon. Insets: Histological appearance of prepsoriatic (symptomless) skin (upper left panel), with unremarkable epidermis and dermis, and rare mononuclear cells present predominantly in the dermis. By contrast, a chronic psoriatic plaque (lower left panel) reveals markedly thickened skin due primarily to accumulation of scale and elongation of rete ridges. In addition, there is loss of the granular cell layer, increased layers of epidermal keratinocytes, and an influx of lymphocytes, DCs, and macrophages into the dermis, accompanied by the presence of dilated and tortuous blood vessels.

spontaneously or after various treatments, can revert back to symptomless or apparently healthy skin, with little or no trace of pre-existing disease activity.

In theories and models advanced to explain psoriatic plaque development, just about every cell type in the lesion has been given center stage. Thus, clinicians and investigators initially focused on the epidermal keratinocyte and viewed psoriatic lesion formation from an "outside-in" perspective (7). Not surprisingly, early therapeutic strategies targeted the hyperplastic epidermis following the lead of oncologists to arrest keratinocyte growth using antiproliferative agents such as arsenic and, later, methotrexate. Other theories implicated psoriatic fibroblasts (8); neutrophils; mast cells (9); nerve cell endings (10); endothelial cells (11); T lymphocytes (12-14), and, specifically, clonal expansion of T cells (15, 16); and DCs (13). The successful use of cyclosporin, which acts to block cytokine release, resulted in a paradigm shift away from epidermal keratinocytes to various immunocyte populations including $\mathrm{T}$ cell subsets and dendritic APCs.

One unifying hypothesis of psoriasis pathophysiology, which assimilated the confederacy of cell types and plethora of soluble mediators, was the cytokine network model (17). In this model, either an exogenously derived stimulus such as trauma, or an endogenous stimulus such as HIV-1, neuropeptides, or ingested medications, was portrayed as triggering a plexus of cellular events by inciting a cascade of cytokines. Initially, this model featured primary pathogenic roles for TNF- $\alpha$ derived from dendritic APCs and keratinocytes, and IFN- $\gamma$ produced by activated Th1type lymphocytes. As presented later, considerable experimental data and therapeutic responses in human subjects support the cytokine network theory of psoriasis. While this theory explains the maintenance of psoriatic plaques via establishment of a vicious cycle, precisely how $\mathrm{T}$ cells are activated in the genesis of psoriatic lesions is unknown. It is also unknown whether the inciting antigen is self-derived (thereby qualifying psoriasis as an autoimmune disease) or is of non-self origin.

Figure 3 presents a working model for the immunopathogenesis of psoriasis, with emphasis on homeostatic versus pathological trafficking patterns of immunocytes. Symptomless skin (left panel), also known as prepsoriatic or uninvolved skin, is not entirely normal, as numerous molecular differences between symptomless skin and healthy skin from non-psoriatic patients are emerging from global gene expression profiling studies (18). Known mediators regulating trafficking of T cells and DCs in symptomless skin are portrayed, including those chemokines and 


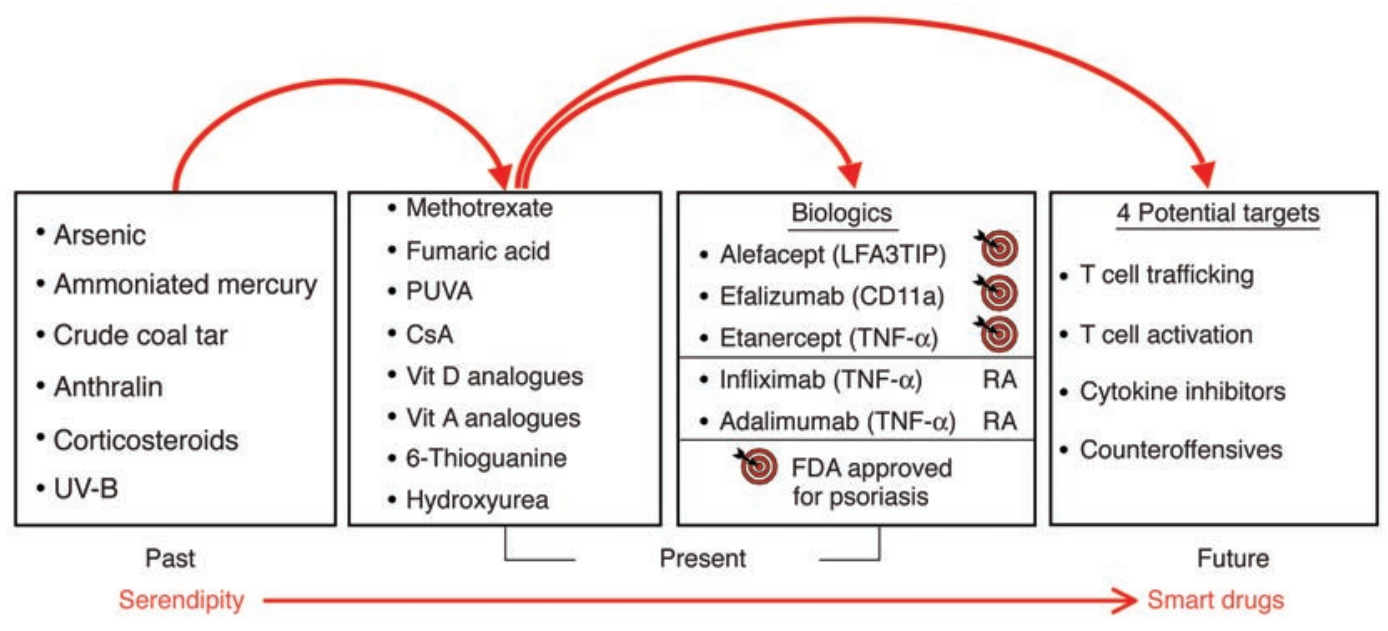

Figure 2

Translational arcs of discovery in psoriasis. In the past, therapeutic agents used by dermatologists were generally discovered serendipitously. Current therapeutic agents are characterized by more specific targeting of defined molecules in the pathological pathways, including, most recently, the use of biologics, which target T cells (alefacept, efalizumab, and etanercept; approved by the US Food and Drug Administration [FDA] for the treatment of psoriasis), and TNF inhibitors (etanercept, infliximab, and adalimumab; approved by the FDA for the treatment of RA). Several agents listed (Past column), including corticosteroids and UV-B light, are still currently used by some dermatologists to treat psoriasis patients. Future drug development should provide additional smart drugs that target specific molecular mediators implicated in the immunopathogenesis of psoriasis. Note: Biologics are defined as therapeutic agents produced by organisms through the use of recombinant biotechnology. CsA, cyclosporin A; PUVA, psoralen and UV-A light therapy; Vit, vitamin.

chemokine receptors that facilitate trafficking to regional lymph nodes. The middle panel of Figure 3 shows acute lesions after a stimulus. A memory $\mathrm{T}$ cell has become activated upon interaction with a dendritic APC. The figure emphasizes costimulatory pathways involving CD2:LFA-3 and LFA-1:ICAM-1. As described in detail in a later section, these molecules participate in formation of an immunological synapse to enhance APC:T cell interactions. Once resident (and recruited) $\mathrm{T}$ cells become activated, a complex network of cytokines, chemokines, and growth factors is created, and the envisioned vicious cycle facilitates further $\mathrm{T}$ cell and DC activation. Ultimately, a chronic plaque is produced (right panel), characterized by intraepidermal $\mathrm{CD}^{+} \mathrm{T}$ cells and neutrophils, and accompanied by epidermal thickening, scale production, and an angiogenic tissue reaction completely remodeling the involved or lesional skin site. We return to Figure 3 in later sections with more detailed molecular analysis to complement the aforementioned emphasis on cellular components and trafficking patterns that contribute to the pathophysiology of psoriasis. In the next section, a review of the genetic approach to understanding psoriasis is presented.

\section{Genetic studies of psoriasis}

Based on pioneering work by Gunnar Lomholt, who explored the relative roles of the environment and heredity in residents of the Faroe Islands (19), and Farber and Nall, who later studied concordance rates in monozygotic twins $(\sim 70 \%)$ and documented kindreds with multiple afflicted family members (20), a search for the genetic basis of psoriasis began. One of the earliest candidate genes for predisposition to psoriasis was the HLA class I allele, specifically HLACw6 (21). A dosage effect of HLA-Cw* 0602 has been observed, where heterozygotes have a relative risk of developing psoriasis of 8.9, compared with 23.1 in homozygous individuals. Also, homozygous individuals experience an earlier onset, but not necessarily a more severe disease course (22). Rapid advancements in human genetic studies have led investigators beyond classical mendelian approaches to use other research methods, including linkage analysis and association-based fine mapping of haplotypes harboring disease-susceptibility alleles with single-nucleotide polymorphism (SNP) analysis. By using genome-wide scans, investigators have mapped (with varying degrees of confidence) at least six different susceptibility loci, designated PSORS1-PSORS6 (23). A list of potentially important loci, including genetic loci that overlap with other chronic inflammatory diseases such as eczema and RA, is provided in Table 1. Several other psoriasis-susceptibility loci have been mapped, including PSORS7 (1p) and PSORS9 (4q31), and additional studies are ongoing by many laboratories $(24,25)$ and the International Psoriasis Genetics Consortium (26).

The major genetic determinant for psoriasis is within the PSORS1 region of the MHC on chromosome $6 \mathrm{p} 21$, as reported by several independent groups, accounting for $30-50 \%$ genetic susceptibility (23). Unfortunately, despite intense study, the definitive gene in this region has not been unequivocally identified. The two most likely candidate loci are HLA-Cw*0602 and the corneodesmosin gene (27), which encodes an adhesive protein expressed by keratinocytes and is important in terminal differentiation of the epidermis, although interpretation is complicated by the extraordinary linkage disequilibrium observed around the MHC. Thus, it is possible that genes such as HLA-Cw6 are in linkage disequilibrium with another gene, or block of genes, at a distinct locus such as killer Ig-like receptor (KIR), as recently reported by Martin et al. (28). KIRs may be important in psoria-

\section{Table 1}

Potential psoriasis-susceptibility loci

\begin{tabular}{lcc} 
Locus name & Approximate location & $\begin{array}{c}\text { Association with other } \\
\text { inflammatory diseases }\end{array}$ \\
PSORS1 & $6 \mathrm{p} 21$ & Asthma \\
PSORS2 & $17 \mathrm{q} 25$ & Eczema \\
PSORS3 & $4 \mathrm{q} 34$ & - \\
PSORS4 & $1 \mathrm{q} 21$ & Eczema \\
PSORS5 & $3 \mathrm{q} 21$ & RA \\
PSORS6 & $19 \mathrm{p} 13$ & - \\
\hline
\end{tabular}




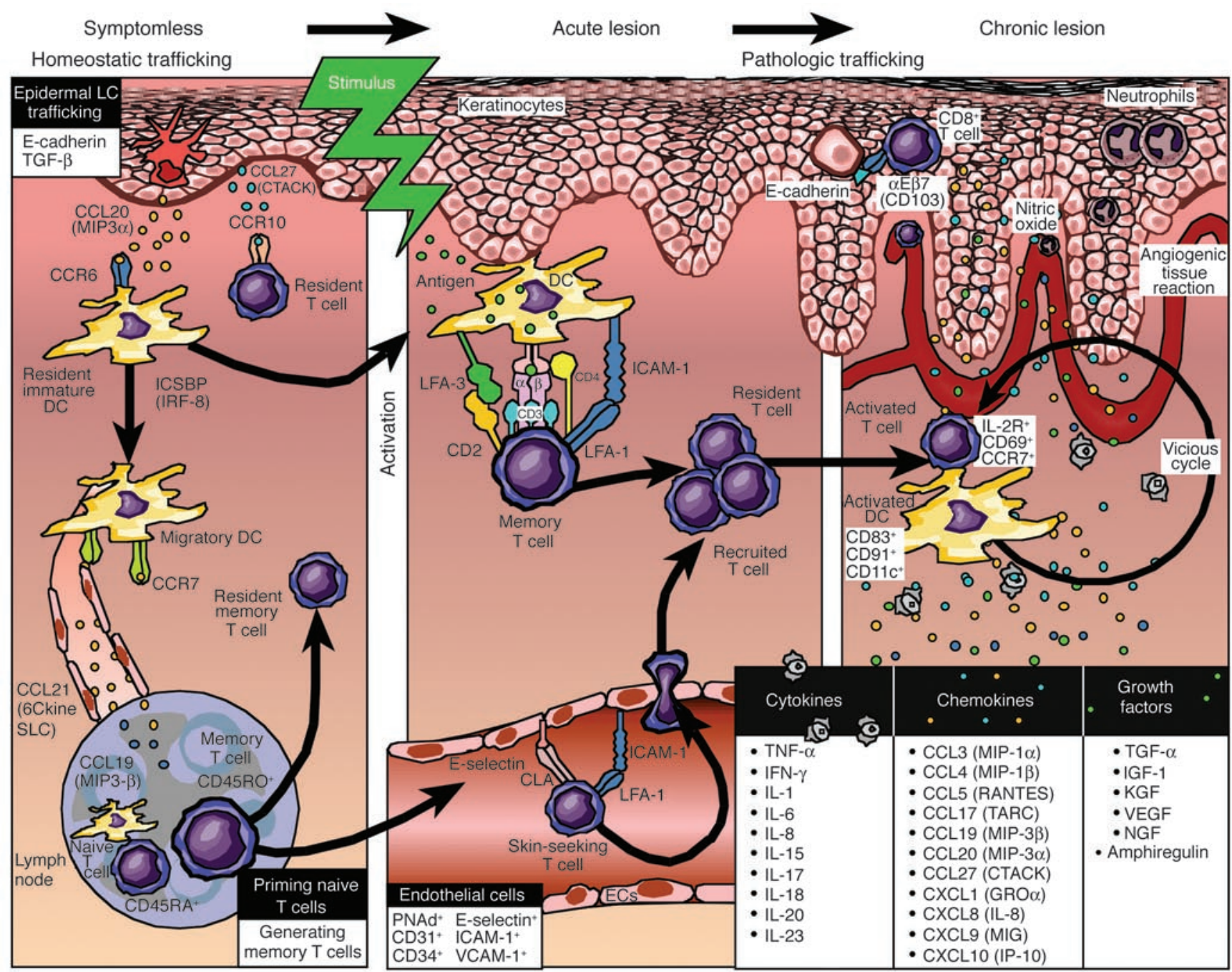

Figure 3

Working model for immunopathogenesis of psoriasis. Multiple stages are proposed for trafficking patterns of immunocytes, involving signals in which symptomless skin is converted into a psoriatic plaque. Symptomless skin is endowed with a confederacy of bone marrow-derived cells, and continuous leukocyte migration between skin and lymph nodes provides immunological vigilance to monitor invading pathogenic organisms. Known mediators of homeostatic trafficking for Langerhans cells (LCs), resident DCs, and T cells are portrayed (left panel). Ideal therapeutic agents for psoriasis should not perturb this physiological process. Following a stimulus, an acute psoriatic lesion forms in which DCs and T cells become activated with formation of an immunological synapse. No consistent antigen has been identified (middle panel). Stimuli may include a danger signal, either an extrinsic, pathogen-associated signal (e.g., pathogen-associated molecules that bind to pattern-recognition Toll-like receptors) or an intrinsic signal derived from within the body (e.g., heat shock proteins that bind to receptors, HIV-1, and ingested medications such as lithium or $\beta$-blockers). Once dendritic APCs and T cells become activated, they release cytokines, chemokines, and growth factors that trigger keratinocyte proliferation, altered differentiation, and an angiogenic tissue response. A vicious cycle of continuous T cell and DC activation can be envisioned within the chronic psoriatic plaque (right panel). A list of relevant cytokines, chemokines, and growth factors that likely conspire with resident and recruited cells to create and sustain psoriatic plaques is provided (lower right panel). Key inflammatory events include intraepidermal trafficking by CD8 ${ }^{+} \mathrm{T}$ cells and neutrophils.

sis (and psoriatic arthritis), since they are expressed on NK cells, as well as NK type T cells (NKT cells), which have been observed in lesional skin and have thus given rise to the consideration of innate immunity in psoriatic lesions (29).

The second most well-characterized disease-susceptibility locus (PSORS2) resides within $17 \mathrm{q} 24-\mathrm{q} 25$. Linkage of psoriasis to this locus has been identified by independent family sets $(30,31)$. Two candidate genes in this region are SLC9A3R1 and NAT9, which are discussed in the following section (32). To understand how these genotyping results may be linked to the immune system and thereby contribute to the immunopathogenesis of psoriasis, it is helpful to review the role of the immunological synapse in psoriasis and autoimmunity, as described in the next section. It should be noted that the field of human molecular genetics and autoimmunity is progressing rapidly, and the initial view of candidate genes is bewildering, yet intriguing. Next, we provide a working model of the immune synapse of $\mathrm{T}$ cells in psoriasis, integrating recent reports describing $\mathrm{T}$ cell signaling pathways in autoimmunity. 


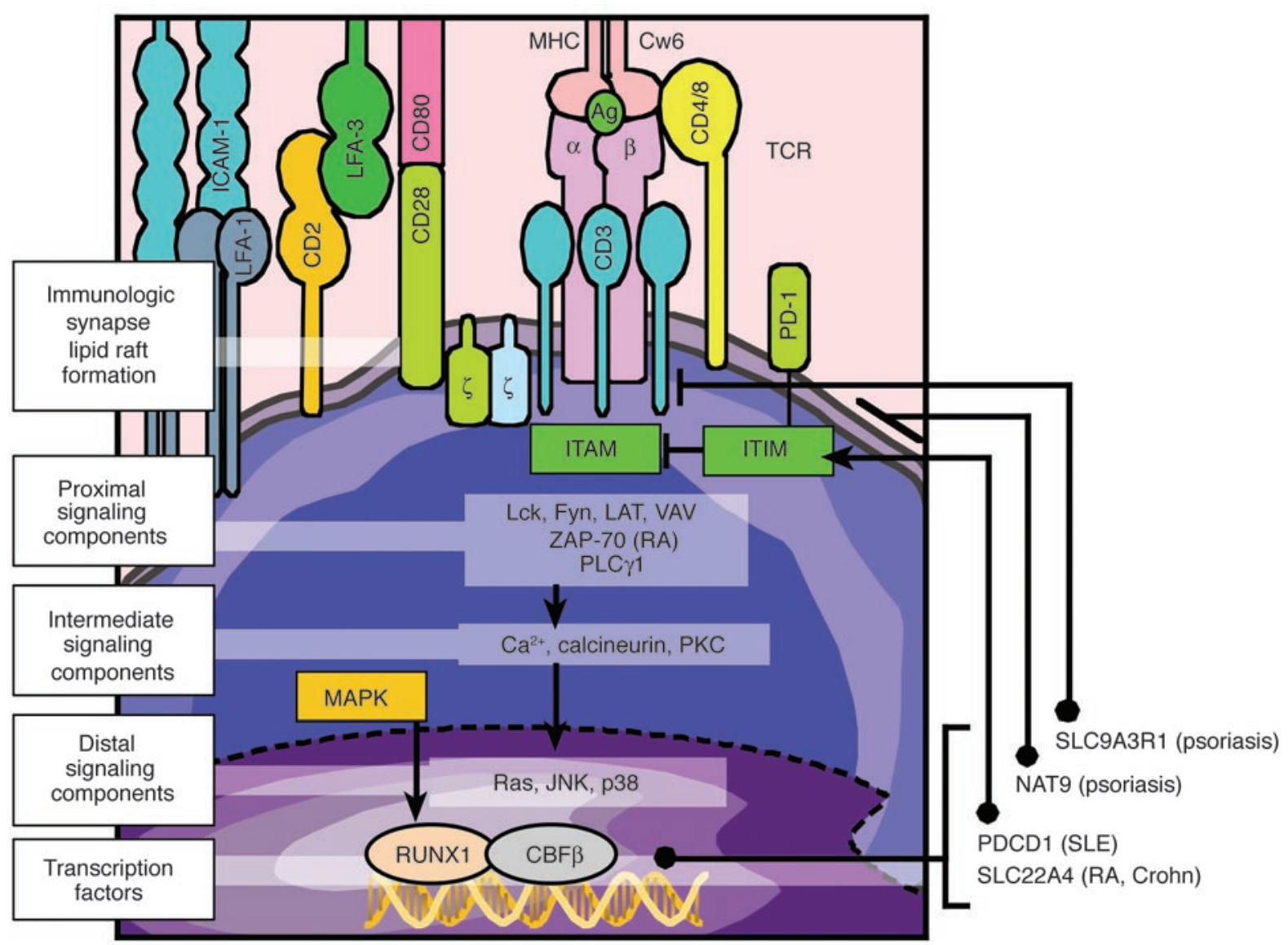

\section{Figure 4}

Immune synapse-related signaling pathway and autoimmune diseases. A possible role for genetic mutations involved in several autoimmune diseases is highlighted, including ZAP-70 (RA); SLC9A3R1 and NAT9 (psoriasis); PDCD1 (systemic lupus erythematosus [SLE]); and SLC22A4 (RA and Crohn disease). T lymphocytes contain both surface receptors and intracellular signaling components that become activated when the TCR is engaged by interactions with an appropriate APC in which antigen is presented in the context of MHC molecules. The APC is displayed in the upper portion of this schematic view. The TCR mediates signaling in conjunction with molecules located in the plasma membrane, including CD3 and $\xi$-chains, which contribute to the formation of an immunological synapse and a lipid raft, leading to activation of proximal, intermediate, and distal signaling components. Several but not all components are portrayed in this figure. Ultimately, transcription factors become activated and bind to respective promoter regions to either enhance or suppress target gene expression. Potential mechanisms linking the genetic findings to functional components of the immunological synapse are shown by solid bold lines.

\section{The immunological synapse and psoriasis}

In mature lymphocytes, $\mathrm{T}$ cell receptor (TCR) signaling is mediated by formation of a multimolecular complex at the $\mathrm{T}$ cellAPC interface, referred to as the immunological synapse (33). The immunological synapse is a discrete cluster of molecules formed between a T cell and an APC that facilitates immune cell interactions. Two major technologies have been used to define events in the immunological synapse: live-cell fluorescent imaging of $\mathrm{T}$ cells in contact with supporting plasma bilayers, and real-time confocal imaging of the T cell-APC interface. Key molecular components include the TCR and, surrounding it, a ring of adhesion molecules, such as LFA-1, which can bind to ICAM-1 expressed by the adjacent cell, e.g., a keratinocyte or APC. The LFA-1 component of the synapse is especially important in psoriasis, as a therapeutic agent (anti-LFA-1 antibody; efalizumab) that blocks this adhesive interaction has recently been approved by the US Food and Drug Administration (FDA) for the treatment of psoriasis (34).
While the immunological synapse controls T cell activation, additional contributory molecules, including other adhesion molecules and costimulatory molecules, also influence $\mathrm{T}$ cell responsiveness. The cell surface molecular pairs CD2:LFA-3 (35) and CD28:CD80/ CD86 (B7.1/B7.2) are examples. Many of these molecules have also been the subjects of clinical trials in psoriatic patients, in which inhibitory reagents have been designed to block T cell activation. For example, an LFA-3 Ig fusion protein (alefacept) has been found to reduce psoriatic lesions (36), and a different fusion protein, CTLA4Ig, which blocks CD28:CD80/CD86 interactions, also improved psoriatic lesions (37). Attempts to modulate $\mathrm{T}$ cells directly in psoriasis include the use of antibodies directed against CD3 (38) and CD4 (39), and a fusion protein containing the cell-binding domain of diphtheria toxin (i.e., denileukin diftitox) has been produced to target and kill activated T cells bearing receptors for IL-2 (40).

For several reasons, the signaling molecules linked to the immunological synapse have become the focus of geneticists interested in defining the molecular basis of autoimmunity (Figure 4). It has 
been suggested that alterations in TCR signaling, which is critical for central tolerance (elimination of autoreactive $\mathrm{T}$ cells), may cause a breakdown in thymic negative selection and hence mediate appearance of autoreactive $\mathrm{T}$ cells (41). Thus, it is possible that psoriasis patients have inherited mutations that block removal of T cells bearing TCRs with a high affinity for self-antigens, such that upon activation, these autoreactive T cells interact with APCs and keratinocytes to create psoriatic plaques. It should be noted that the field involving the molecular basis that regulates formation of the mature $\mathrm{T}$ cell repertoire is complex and rapidly changing, so caution is warranted in ascribing specific roles to the immunological synapse and TCR signaling as regards negative selection in human subjects. Nonetheless, to gain insights into this possible scenario, it is useful to review progress in other autoimmune diseases, beginning with mutations in the proximal components of the TCR signaling pathway (e.g., ZAP-70). Recently, Sakaguchi et al. (42) reported a strain of mice that spontaneously develop a chronic systemic inflammatory disease resembling RA. CD4 ${ }^{+} \mathrm{T}$ cells bearing a point mutation in a gene encoding an $\mathrm{SH} 2$ domain of ZAP-70, a key upstream signal-transducing molecule, could transfer the disease state in a variety of different mouse strains. Clinical manifestations in this model were also influenced by environmental conditions, including a susceptibility locus in the MHC complex that is analogous to that for psoriasis (PSORS1 and PSORS2). In preliminary studies, this group also identified heterozygous mutations in the immunoreceptor tyrosine-based activating motif(ITAM) region of the TCR- $\zeta$ chain in $2.5 \%$ of RA patients, which is relevant because of the physical association between ITAM and ZAP-70.

Three recent reports also merit consideration, as they involve polymorphisms in regulatory (promoter) regions of genes that impact proper immune synapse function with emergence of autoreactive T cells (43). In Nordic multicase families with systemic lupus erythematosus (SLE), the strongest candidate for disease association was found to be within the programmed cell death 1 gene (PDCD1) (44). The associated allele of the SNP altered a binding site for runt-related transcription factor 1 (RUNX1). RUNX1 (also known as AML1) belongs to a family of transcription factors that can either inhibit or promote expression of a variety of genes. The transactivating potential of RUNX1 can be enhanced by phosphorylation events mediated by the MAPK pathway. Since PDCD1 encodes a protein (PD-1) with an immunoreceptor tyrosine-based inhibitory motif (ITIM), which could thereby inhibit autoreactive $\mathrm{T}$ cells (i.e., participate in peripheral tolerance), deletion of RUNX1-binding sites may contribute to aberrant regulation of PDCD1, leading to dysregulated self-tolerance and persistent lymphocyte activation.

Besides identification of disease-associated regulatory singlenucleotide polymorphisms (rSNPs) in RUNX-binding sites for lupus, other groups identified rSNPs in psoriasis and RA patients $(31,45)$. In psoriatic patients, the rSNPs of interest may be regulating expression of two separate genes - SLC9A3R1 and NAT9 (32). SLC9A3R1 (solute carrier family 9, isoform 3 regulating factor 1 ) is a PD-2 domain-containing phosphoprotein that associates with members of the ezrin-radixin-moesin family. It can negatively regulate immune synapse function, influencing negative selection during development (central tolerance) and thus leading to the emergence of autoreactive T cells (46), or alter local immune responses in the skin with inappropriate or dysregulated $\mathrm{T}$ cell activation. Similarly, NAT9, a new member of the $N$-acetyltransferase family, alters glycosylation patterns of various immuno- regulatory proteins, including MHC class I, and components of the immunological synapse. In RA patients, the rSNP may regulate expression of SLC22A4 (solute carrier family 22, isoform 4), which is an organic cationic transporter. The location of this gene is $5 q 31$, a locus also implicated in Crohn disease (47). While this locus contains a cytokine gene cluster, SLC22A4 is not a cytokine, and its specific immunoregulatory role or impact on inflammatory processes is currently unclear. While this specific organic cation transporter does not appear to regulate uptake of L-arginine (48), there is growing interest in the importance of cationic amino acid transporter expression in skin diseases. If SLC22A4 were found to influence L-arginine levels, this could, in turn, modulate extracellular levels of nitric oxide and thereby alter TCR- $\zeta$ chain expression (49), providing a further link between the genetics of autoimmunity and the immunological synapse. Before finishing this brief review of ionic transporters, it should be noted that a different family of cation/chloride cotransporters has also been implicated in psoriasis (PSORS5) (50).

As can be appreciated from these new clinical trial results and DNA sequencing/genotyping data, advances related to nonantigen-specific (i.e., costimulatory) pathways have outpaced attempts to define or identify a precise antigen in this T celldependent skin disease. Unfortunately, no consistent antigen has been identified, nor has a specific gene been validated, as a definitive cause of psoriasis. Perhaps more gratifying for investigators have been the consensus and progress in defining the cytokine network operative in psoriatic lesions. Indeed, as described next, delineation of cytokine cascades facilitated transition in the clinical arena from serendipity to more selective and highly targeted therapeutics developed specifically for psoriasis.

\section{Molecular effector pathways in psoriasis}

Figure 3 provides our working hypothesis for the immunopathogenesis of psoriasis. This model contains elements from earlier proposals $(13,17)$. Multiple signaling pathways are envisioned to contribute to the pathological process whereby symptomless skin is converted to psoriatic plaques. An initial activating signal is portrayed as perturbing epidermal keratinocytes (13). Mild skin trauma, such as a cut or abrasion, in which epidermal keratinocytes are damaged can trigger psoriasis (this is known as the Köbner phenomenon) and a change in the epidermal maturation pathway. Exactly what happens in keratinocytes to create a "danger signal" is unclear (51), but several possible molecular events may occur to activate resting dendritic APCs (52), followed by delivery of an antigenic signal to $\mathrm{T}$ cells (so-called signal no. 1). These possible events include perturbation of the barrier function of skin with release of preformed or rapidly produced cytokines such as IL-1 and TNF- $\alpha$ (53); exposure of DCs constitutively expressing MHC class II molecules such as HLA-DR in the epidermis and/or dermis to bacterial products from skin flora (54), including superantigens (55); release of heat shock proteins from epidermal keratinocytes that could bind to CD91 expressed by dendritic APCs (56); exposure of keratinocytes and dendritic APCs to glycolipids that bind to CD1d $(57,58)$; and engagement of Toll-like receptors on dendritic APCs by unspecified molecular determinants (59).

The next step involves delivery of additional costimulatory signals (signal no. 2), which is likely to involve CD28 and B7 family members including B7.1 (CD80) and B7.2 (CD86) based on in vitro and in vivo studies $(37,60)$. New B7-related family members 
(and their ligands) have been identified, and it will be interesting to determine whether they also play a role in the pathogenesis of psoriasis. Once T cells and dendritic APCs are fully activated, they can create a "cytokine storm" composed of numerous cytokines, chemokines, and growth factors, as summarized in the lower right panel of Figure 3. A vicious cycle can then be envisioned in which keratinocytes, endothelial cells, neutrophils, and immunocytes in the vicinity become activated and conspire in the creation of a psoriatic plaque. Macrophages are also present, and it remains to be determined whether they are functioning to enhance the inflammatory response or to limit local immune reactions.

One of the first questions addressed by investigators was whether a polarized Th1- versus Th2-type cytokine-production profile would apply to human diseases. Initial analysis of psoriatic plaques with rather limited cytokine analysis revealed a Th1-type profile $(61,62)$. IFN- $\gamma$, TNF- $\alpha$, and IL-12, but not IL-4, IL-5, or IL-10, were documented within psoriatic plaques at the mRNA and protein levels. As investigators probed the interactions among the Th1type cytokines in vitro, it became apparent that there was synergy between IFN- $\gamma$ and TNF- $\alpha$ with regard to production of adhesion molecules such as ICAM-1, and chemotactic polypeptides such as IL-8 or monocyte chemotactic activating factor-1 (MCAF-1). The challenging task of integrating the in vitro and in vivo findings into a coherent cytokine network that had spatial and temporal validity was first undertaken in 1991 (17). This initial portrayal of the cytokine network in psoriasis placed TNF- $\alpha$ at center stage as a key primary cytokine involved in the induction and maintenance of plaques. Polymorphisms in genes regulating cytokine production have been identified for TNF- $\alpha$ and other inflammatory mediators $(63,64)$. Given pleiotropic effects by which TNF- $\alpha$ influences a wide variety of cell types in both skin and joints of psoriatic patients, several pharmaceutical companies began targeting this cytokine, using two approaches. However, before we enter the therapeutic arena, additional details of the cytokine network are presented.

During the past decade, numerous reports have provided additional molecular details concerning the cytokine network in psoriatic plaques including chemokines, growth factors, and signal transduction pathways. With the advent of high-throughput cDNA-based microarrays, this list has grown exponentially $(65,66)$. Space constraints do not permit a complete summary of all of these mediators; however, some of the most prominent components are listed in Figure 3. Besides these mediators, consideration of highmobility group B1 (HMGB1) protein binding to its receptor, receptor for advanced-glycation end products (RAGE) (65), is warranted, since HMGB1 influences cytokines such as TNF- $\alpha$. In other chronic inflammatory diseases, HMGB1 is also considered an important regulator (67). Production of cytokines derived from APCs includes TNF- $\alpha(68)$ and IL-23 $(69,70)$. T cells are the likely source for IFN- $\gamma$, IL-15 $(71,72)$, and IL-17 (73), whereas keratinocytes can produce IL-1, IL-6, and IL-8, as well as IL-18 (74) and IL-20 (75). Besides these cytokines, numerous chemokines and chemokine receptors are present in psoriatic plaques. When chemokines bind to their respective receptors, they activate the cells, which may be important not only for recruitment into the skin, but also for their local release of cytokines and growth factors.

Chemokines and chemokine receptors of interest in the immunopathogenesis of psoriasis include TARC (CCL17), MIG (CXCL9), IP10 (CXCL10), MDC (CCL22), and RANTES (CCL5), as recently reviewed by Krueger (4), as well as CXCR2, CXCR3, CCR4, CCL27-CCR10, MIP3 $\alpha$ (CCL20), MIP3 $\beta$ (CCL19), and
CCR6. In addition, nitric oxide is present, which may contribute to an angiogenic tissue reaction, accompanied by many growth factors present at elevated levels within psoriatic plaques, including TGF- $\alpha$, IGF-1, keratinocyte growth factor (KGF), VEGF, nerve growth factor (NGF), amphiregulin, and IL-20 (4). Given the plethora of these cytokines, chemokines, and growth factors, it should not be surprising that the end result is a thick, erythematous scaly plaque. In general, activated $\mathrm{CD}^{+} \mathrm{T}$ cells are primarily located in the dermis and $\mathrm{CD}^{+} \mathrm{T}$ cells in the psoriatic epidermis, accompanied by tangled collections of dendritic APCs predominantly located in the dermis.

Besides those molecules listed, potentially important signaling pathways including NF- $\mathrm{B}$, STAT-1, STAT- 3 , and IFN- $\alpha$-inducible proteins are also now on the radar screen of psoriasis research $(65,66)$. Not only can high-throughput cDNA-based technology be used to identify specific transcripts that are elevated in psoriatic plaques; sequential analysis of plaques following treatment can be completed to explore the pharmacogenomics of psoriasis (65). Knowing which transcripts are reduced as lesions improve provides potential new therapeutic targets for future clinical trials, as detailed in the last section of this review. In the next section, additional lessons regarding the immunopathogenesis of psoriasis, derived from two different animal models, are reviewed. Besides xenogeneic animal models, transgenic murine models are important, as exemplified by a recent report in which genetic inactivation of a chemokine receptor 2 unexpectedly provoked a severe disease state similar to human RA, rather than the predicted reduction in inflammation (76).

\section{Lessons learned from xenogeneic animal models}

Up until the introduction of cyclosporin A, the prevailing dogma in dermatology was that psoriasis fundamentally represented a disease of epidermal keratinocytes (77). Early clinical evidence implicating bone marrow-derived immunocytes as primary pathogenic cells emanated from bone marrow transplantations. Formal proof that psoriasis could be transferred by immunocytes required use of animal models in which human skin was engrafted onto SCID mice $(78,79)$. SCID mice are a strain with introduced mutations that render them genetically incapable of producing either $\mathrm{T}$ cells or B cells but retain their capacity to produce neutrophils and NK cells. Hence, they cannot reject human skin grafts and became widely used for organ-engraftment studies. It should be noted that psoriasis appears to be a uniquely human disease, as it has never been observed in primates or other animals. Moreover, given the likelihood that psoriasis is polygenic, attempts to develop transgenic rodent models with single-gene mutations or deletions have failed to generate skin lesions with all the relevant clinical, histological, and immunophenotypic abnormalities.

The ability to create bona fide psoriatic plaques using human skin grafted onto SCID mice opened up new avenues of investigation. Two important sets of observations will be highlighted. First, these new in vivo-based tools allowed researchers to determine whether pathogenic $\mathrm{T}$ cell subsets included $\mathrm{CD}^{+}$or $\mathrm{CD}^{+} \mathrm{T}$ cells, and whether other T cell subsets, such as NKT cells, may be relevant to psoriasis. The initial observation in which prepsoriatic skin was converted to psoriatic plaques using the SCID-Hu model suggested that CD4 ${ }^{+}$ $\mathrm{T}$ cells, but not $\mathrm{CD}^{+} \mathrm{T}$ cells, were capable of triggering psoriasis (80). A second group, using a pure murine inflammatory skin model supported this conclusion (81). Results implicating $\mathrm{CD}^{+} \mathrm{T}$ cells were unexpected, as the genetic data overwhelmingly implicated 
A

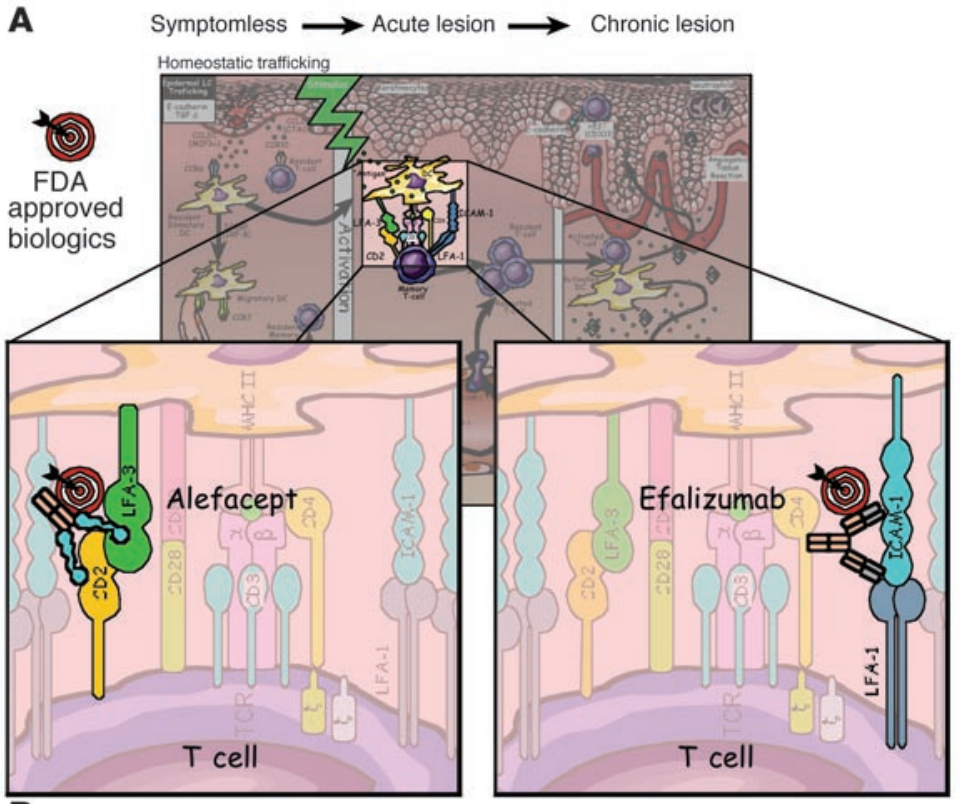

B
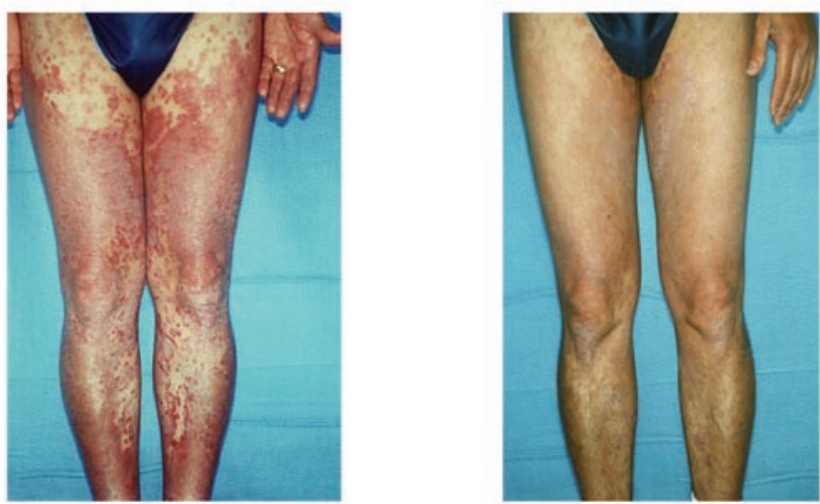

\section{Figure 5}

(A) T cell-targeted therapies in psoriasis. Two FDAapproved biologics (alefacept and efalizumab) are portrayed; the molecular target is identified on the T cell surface (upper panels). Note that the site of action of these agents is depicted in the skin, but the therapeutic efficacy may include other anatomical sites such as lymph nodes or other secondary lymphoid tissues. Alefacept targets the CD2:LFA-3 ligand/receptor interactions, whereas efalizumab targets the LFA-1:ICAM-1 ligand/receptor pair of surface molecules expressed by $T$ cells and APCs, respectively. (B) The clinical response of a patient with severe psoriasis (left) to efalizumab administered for 2 months. Note the almost complete clearing of lesions on the lower extremities and hand (right).
HLA-Cw6 $(21,23)$, an MHC class I molecule usually linked to CD8 ${ }^{+}$ $\mathrm{T}$ cells rather than $\mathrm{CD} 4^{+} \mathrm{T}$ cells. This surprising result led to a search for other surface receptors on $\mathrm{CD}^{+} \mathrm{T}$ cells that can recognize $\mathrm{MHC}$ class I molecules such as molecules shared by NK cells (so-called NKT cells). Indeed, two independent groups have now found evidence for a possible role of NKT cells in the immunopathogenesis of psoriasis $(82,83)$. However, the precise identity of the actual pathogenic $\mathrm{T}$ cell is not currently known. Intraepidermal CD8 ${ }^{+} \mathrm{T}$ cells, which are activated and present in psoriatic plaques, might actually be the true pathogenic T cell, particularly since HLA-Cw6 remains the strongest genetic candidate locus for psoriasis susceptibility.

The second set of observations related to use of the SCID-Hu model has shown that the model is useful as a pharmacologically validated experimental tool for preclinical screening of promising therapeutic agents. Numerous reports have now described the favorable responses of engrafted psoriatic plaques to a wide range of agents targeting a variety of putative molecular mediators $(72,84-86)$. Not only can the SCID-Hu model assist the pharmaceutical and biotechnology industries, but response of grafted human skin to the targeted pathways will also provide valuable new insights into the relevance of specific molecules in maintaining the psoriatic phenotype. Such new insights are unlikely to be achieved by examination of various cell types only in tissue culture, or by the use of transgenic rodents.
Besides the SCID-Hu xenogeneic model, a new and different approach has recently been described in which psoriatic plaques are spontaneously created. This animal model uses AGR129 mice (which are deficient in type I and type II IFN receptors) and has recombination-activating gene 2 knocked out (87). When symptomless skin is engrafted onto AGR129 mice, plaques spontaneously develop without exogenous delivery of $\mathrm{CD} 4^{+} \mathrm{T}$ cells. Moreover, when human skin is serially examined after transplantation, resident human $\mathrm{T}$ cells undergo local proliferation with production of TNF- $\alpha$. Such T cell proliferation and cytokine release are crucial for development of a psoriatic phenotype, since selective agents used to block $\mathrm{T}$ cell proliferation (anti-human CD3 antibody) or TNF inhibitors (infliximab or etanercept) prevent conversion of prepsoriatic skin to psoriatic plaques.

Taken together, these results warrant a paradigm shift in which resident $T$ cells and APCs are viewed as necessary and sufficient for induction of psoriasis. The precise mechanism underlying resident $\mathrm{T}$ cell proliferation and cytokine release is unclear, but AGR129 mice differ from SCID mice in that they lack NK cells. Thus, AGR129 mice may serve as a host that cannot reject human CD4 ${ }^{+}$ $\mathrm{T}$ cells or DCs in the graft by virtue of the absence of NK cells. Furthermore, the lack of IFN receptors in AGR129 mice may facilitate a cytokine milieu (including IL-7 or IL-23) conducive to activation 
and proliferation of dormant pathogenic $\mathrm{T}$ cells residing in the prepsoriatic skin. Before conclusion of this section, three additional points should be emphasized. First, the AGR129 results support a key pathogenic role for TNF- $\alpha$ in the creation of plaques. Second, these results nicely complement earlier results using SCID mice in which engraftment of psoriatic plaques revealed a sustained phenotype after transplantation, indicating that immunocytes contained within the plaque were necessary and sufficient for maintenance of the chronic inflammatory pathological process. Third, to our knowledge, the AGR129 mouse model represents the first spontaneous animal model for a human autoimmune disease process, and it will facilitate development and testing of agents that could be used to prevent, rather than simply reverse, psoriatic lesions.

However, there are limitations in using xenograft models. First, engrafted skin has been disconnected from its lymphatic drainage, and, upon engraftment onto mice, no further human bone marrow-derived cells can participate in lesion formation; this precludes studies of trafficking between circulating cells and skin. Second, the models are cumbersome and require availability of patients willing to donate keratome samples from their skin. Third, the surgical procedure used to procure tissue, as well as the transplantation process, may produce wound-healing responses that complicate interpretation of the results. Next, a review of exciting new clinical developments that improve psoriatic skin lesions and psoriatic arthritis is presented.

\section{Immunological targeted therapy in psoriasis}

Because of space limitations, this section is not a complete review of all agents used or tested for management of psoriatic patients; it focuses instead on $\mathrm{T}$ cell-targeted treatments recently approved by the FDA, and those agents that target cytokines. Figure 5 profiles two different $T$ cell-targeted therapies approved by the FDA. Alefacept is an LFA-3 Ig fusion protein that interferes with CD2: LFA-3 interactions (upper left panel). Efalizumab is an anti-LFA-1 antibody that interferes with LFA-1:ICAM-1 interactions, which also improves psoriatic lesions (upper right and lower panels). Besides these T cell-targeted therapies, three main cytokine-targeted approaches have been successfully used in the treatment of RA (Figure 2), psoriatic arthritis (4), and psoriatic skin lesions.

In the first approach, small molecules were developed that prevent cytokine release from immunocytes. Initial success was obtained using cyclosporin A, which is a calcineurin inhibitor that blocks signal transduction pathways that lead to cytokine release. Another calcineurin inhibitor is tacrolimus, which is 50-100 times more potent than cyclosporin A (88). Other immunosuppressant drugs that block cytokine release and have been found to be effective in psoriasis include sirolimus (rapamycin) (89) and pimecrolimus (ASM981) (90). These agents are derived from various soil fungi, contrasting sharply with a new wave of biological agents produced using recombinant DNA technology.

The other two strategies, based on use of biotechnology, attempt to neutralize cytokines once they are produced. Several agents target TNF- $\alpha$, although many other approaches that target different cytokines such as IFN- $\gamma$ and the p40 subunit shared by IL-12 and IL-23 are under active study. One strategy, using recombinant biotechnology to neutralize TNF- $\alpha$, is to produce highly specific mAb's that bind TNF- $\alpha$, thereby preventing it from interacting with its surface receptors. Examples of such neutralizing antibodies against TNF- $\alpha$ include both chimeric antibodies (proteins containing a predominantly human backbone sequence combined with murine amino acid sequences), such as infliximab (91), and the fully humanized antibody adalimumab (92). The second strategy produces soluble receptors lacking signaling domains so as to bind and thereby neutralize TNF- $\alpha$. This strategy derived from consideration of how viruses escape immunosurveillance using soluble receptors. Examples of soluble receptors are etanercept, which binds TNF- $\alpha$ and improves psoriatic arthritis and skin lesions (93, 94); and onercept, which improves Crohn disease (95).

Not only do many of the aforementioned treatments targeting TNF- $\alpha$ clearly benefit the majority of patients with psoriatic skin lesions, but they also can improve the inflammatory process in psoriatic joints, as well as in patients with RA and inflammatory bowel diseases such as Crohn disease. However, not all of these drugs work equally well in all patient groups, nor does the same drug necessarily improve both skin and joint manifestations equally. Also, since these drugs require injections for systemic delivery, there are injection-site reactions and concerns about potential infectious complications and oncological issues that will require long-term follow-up and clinical vigilance.

Finally, as a derivative of the second biotechnology approach, i.e., counterattack of existing cytokines, investigators are also producing recombinant human Th2 cytokines, and administering them to try to nullify the Th1-type cytokines or facilitate selective cytokine skewing of pathogenic T cells. Examples include delivery of IL-10 (96), IL-11 $(65,97)$, or IL-4 (98). While anti-inflammatory effects of IL-10 and IL-11 were relatively modest compared with those of the TNF- $\alpha$ inhibitors, clearly the entire field is rapidly moving from nonspecific treatments such as arsenic, corticosteroids, methotrexate, and UV light to more selective treatments aimed at defined pathogenic targets for patients with psoriasis and other chronic inflammatory diseases.

\section{Future directions}

There is no question that advances in understanding the cellular immunology and biology of psoriasis, when coupled with the biotechnology revolution and rapid advances derived from human genetic studies of autoimmunity, have enhanced insights into the cause and treatment of psoriasis. However, we still lack a cure for this common and enigmatic disease, and we have not unequivocally identified genes or antigens responsible for its occurrence worldwide. Moreover, many patients still experience significant side effects from currently available treatments, including development of skin cancers in nonlesional skin (with psoralen and UV-A light therapy), abnormal renal function (with cyclosporin A), and liver abnormalities (with methotrexate), to name a few.

As should be evident from this review, these are exciting times for clinicians and scientists interested in chronic inflammatory diseases such as psoriasis. Figure 6 emphasizes that there are still new innovations worth considering in psoriasis research. Future potential targets include $\mathrm{T}$ cell trafficking, $\mathrm{T}$ cell activation, cytokine inhibitors, and counteroffensive strategies. Briefly, trafficking targets may include very late antigen-1 (VLA-1, or $\alpha_{1} \beta_{1}$ integrin) expressed by $\mathrm{T}$ cells, which mediates binding to ECM molecules including collagen I and IV. Antibody against VLA-1 inhibits cutaneous hypersensitivity and arthritis in animal models (99). Another trafficking-related target is VLA-4, which recognizes VCAM-1, and antibodies against VLA-4 improve multiple sclerosis and Crohn disease (100). T cell activation may also be impacted by these antibodies against surface molecules, as well as by other agents, that target various components of the immu- 


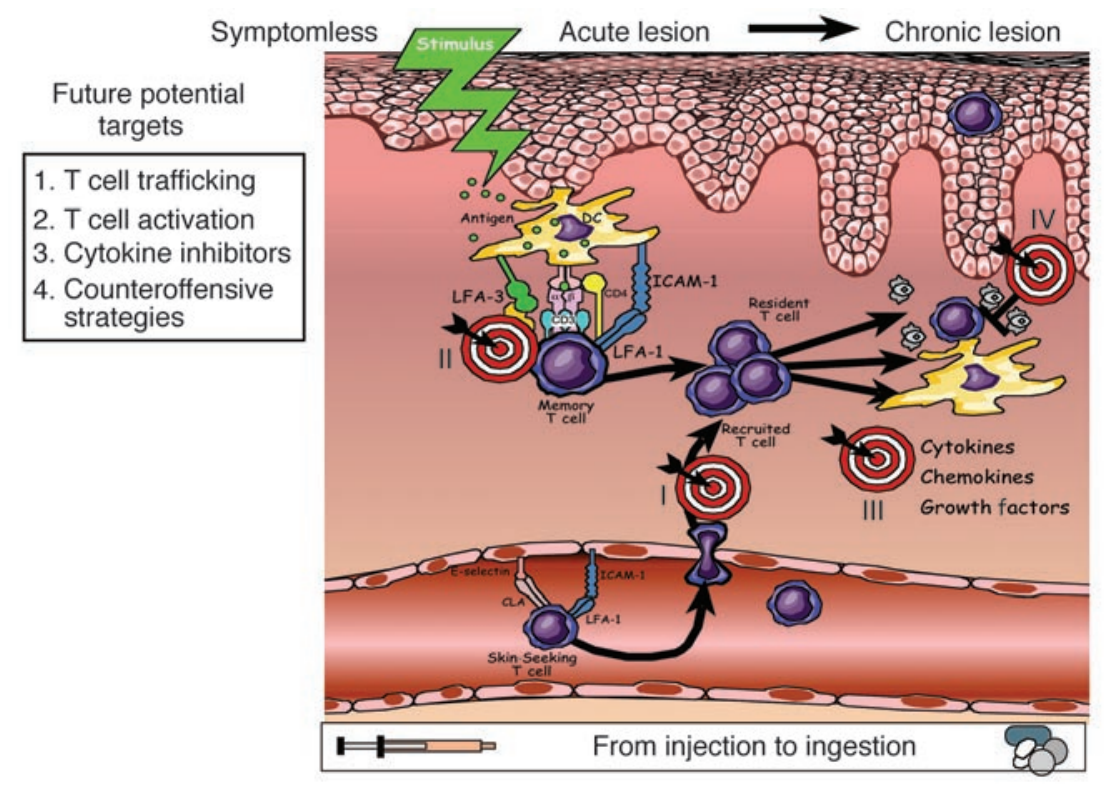

\section{Figure 6}

Innovations in psoriasis therapy. Future potential targets in the treatment of psoriasis are depicted. These therapeutic opportunities can be considered in the context of four broad areas: (I) T cell trafficking, (II) T cell activation, (III) cytokine inhibitors, and (IV) counteroffensive strategies. To enhance patient compliance and safety, antiinflammatory and next-generation immunosuppressants ideally would be able to be ingested rather than injected. nological synapse depicted in Figure 4. Given the remarkable success of several different agents that target TNF- $\alpha$ in psoriasis, it is likely that additional reagents aimed at other cytokines, as described below, will surface in dermatology clinics. In the category of counteroffensive strategies, it may be possible in the near future to enhance relative levels for regulatory $\mathrm{T}$ cell subsets to negate the pathogenic $T$ cell subsets.

Future challenges are severalfold and include the following: First, carefully monitor patients on currently approved new biologics and determine the long-term sequelae of chronic inflammatory inhibitors as regards potential risks of developing infections and neoplasms. Second, continue to define and refine the cytokine network working both upstream and downstream from TNF- $\alpha$. In other words, identify what specific triggers account for the initial production of TNF- $\alpha$. Such studies are likely to consider both innate and adaptive immunity. Cytokines that may impact TNF- $\alpha$ production include HMGB1, IL-15, and IL-23, to name a few. Third, elucidate the genetic basis by which this disease is transmitted from generation to generation. Our colleagues investigating Crohn disease have provided an encouraging precedent in which specific mutations in the NOD2 gene are implicated in a subset of patients with this chronic inflammatory disease $(101,102)$. At least one group has identified a mutation in the NOD2 gene (also known as CARD15) that confers susceptibility to psoriatic arthritis (103). Originally, it was believed that NOD2 was primarily expressed in monocytes and macrophages, but more recent studies indicate that NOD2 is also present in specialized epithelial cells known as Paneth cells, which are most numerous in the terminal ileum (104). Such new insights require reconsideration of an important role for epithelial cells in the immunopathogenesis of psoriasis. It would be ironic if the quest to identify the key cell in psoriasis, begun with a focus on keratinocytes, were concluded by studies aimed at keratinocytes once again.

Regarding the genetics of psoriasis, it remains to be determined whether final elucidation will reveal a rare gene variant for a common disease (as seen in Crohn disease), or whether this apparently complex genetic trait is created by multiple common low-risk susceptibility genes. It is intriguing to consider the possibility that mutations responsible for psoriasis may impact negative selection, thereby facilitating emergence of autoreactive $\mathrm{T}$ cells. Since only $5 \%$ of developing thymocytes emerge as mature $\mathrm{T}$ cells, ridding the immune system of autoreactive $\mathrm{T}$ cells may be imperfect, and tantalizing clues are beginning to point to this possibility for psoriasis and other autoimmune diseases, as highlighted in this review (Figure 4).

The fourth and final challenge for the future, based on the AGR129 mouse xenograft studies, is to focus on other resident cells besides keratinocytes, particularly localized subsets of immunocytes. Similarly, new clinical studies point to consideration of topical approaches targeting resident skin cells that may be associated with less systemic adverse events if the psoriatic patient does not have concomitant joint involvement. Besides novel transdermal delivery systems, the next generation of targeted therapy could include use of statins that interfere with LFA-1 (105), or novel small molecules that can interfere with inflammatory responses mediated by p38 signaling (106), cytokine receptors (107), or rationally designed dominant negative TNF- $\alpha$ variants (108).

Ultimately, once specific targets are validated with injectable reagents, such as the biological reagents, it will be possible to design orally available drugs to enhance patient compliance, safety, and medical costs. For example, orally ingestible statins that interfere with LFA-1 signaling should improve psoriasis, given the clinical efficacy of the anti-LFA-1 antibody (efalizumab). Perhaps lessons learned from studies in psoriasis and the skin immune system will also pay dividends for patients with other cytokine-mediated chronic inflammatory autoimmune diseases, including RA, lupus erythematosus, and inflammatory bowel disorders.

\section{Acknowledgments}

The authors appreciate the assistance of Stephanie Hiffman for manuscript and figure preparation. Brian Bonish created original artwork. The NIH has provided 13 years of continuous funding to Brian J. Nickoloff to support laboratory studies of psoriasis (NIH R01 AR40065). June Robinson provided a clinical photograph (Figure 1), and James Krueger provided before/after clinical 
photographs (Figure 5). We also thank Pamela Pollock, Barbara Osborne, Kenneth Gordon, Jonathan Barker, and Jonathan Curry for reviewing the manuscript.

The authors apologize for omitting numerous important references because of space constraints. However, a list of over 200 relevant citations, arranged alphabetically by author, is available at http:// www.jci.org/cgi/content/full/113/12/1664/DC1 to properly credit the other research groups responsible for data and concepts mentioned in the review.

Address correspondence to: Brian J. Nickoloff, Skin Disease Research Laboratory, Cardinal Bernardin Cancer Center, Loyola University of Chicago, 2160 S. First Avenue, Building 112, Room 301, Maywood, Illinois 60153, USA. Phone: (708) 327-3241; Fax: (708) 327-3239; E-mail: bnickol@lumc.edu.
1. Krueger, G., et al. 2001. The impact of psoriasis on quality of life: results of a 1998 National Psoriasis Foundation patient-membership survey. Arch. Dermatol. 137:280-284.

2. Bhalerao, J., and Bowcock, A.M. 1998. The genetics of psoriasis: a complex disorder of the skin and immune system. Hum. Mol. Genet. 7:1537-1545.

3. Henseler, T., and Christophers, E. 1985. Psoriasis of early and late onset: characterization of two types of psoriasis vulgaris. J. Am. Acad. Dermatol. 13:450-456.

4. Krueger, J.G. 2002. The immunologic basis for the treatment of psoriasis with new biologic agents. J. Am. Acad. Dermatol. 46:1-23; quiz 23-26.

5. Nickoloff, B.J. 2001. Creation of psoriatic plaques: the ultimate tumor suppressor pathway. A new model for an ancient T-cell-mediated skin disease. Viewpoint. J. Cutan. Pathol. 28:57-64.

6. Henseler, T., and Christophers, E. 1995. Disease concomitance in psoriasis. J. Am. Acad. Dermatol. 32:982-986.

7. Barker, J.N. 1991. The pathophysiology of psoriasis. Lancet. 338:227-230.

8. Saiag, P., Coulomb, B., Lebreton, C., Bell, E., and Dubertret, L. 1985. Psoriatic fibroblasts induce hyperproliferation of normal keratinocytes in a skin equivalent model in vitro. Science. 230:669-672.

9. Ackermann, L., et al. 1999. Mast cells in psoriatic skin are strongly positive for interferon-gamma. Br. J. Dermatol. 140:624-633.

10. Raychaudhuri, S.P., Rein, G., and Farber, E.M. 1995. Neuropathogenesis and neuropharmacology of psoriasis. Int. J. Dermatol. 34:685-693.

11. Lowe, P.M., et al. 1995. The endothelium in psoriasis. Br. J. Dermatol. 132:497-505.

12. Gottlieb, A.B. 1988. Immunologic mechanisms in psoriasis. J. Am. Acad. Dermatol. 18:1376-1380.

13. Nickoloff, B.J. 1999. The immunologic and genetic basis of psoriasis. Arch. Dermatol. 135:1104-1110.

14. Valdimarsson, H., Baker, B.S., Jonsdottir, I., Powles, A., and Fry, L. 1995. Psoriasis: a T-cell-mediated autoimmune disease induced by streptococcal superantigens? Immunol. Today. 16:145-149.

15. Chang, J.C., et al. 1997. Persistence of T-cell clones in psoriatic lesions. Arch. Dermatol. 133:703-708.

16. Vollmer, S., Menssen, A., and Prinz, J.C. 2001. Dominant lesional $\mathrm{T}$ cell receptor rearrangements persist in relapsing psoriasis but are absent from nonlesional skin: evidence for a stable antigen-specific pathogenic $\mathrm{T}$ cell response in psoriasis vulgaris. J. Invest. Dermatol. 117:1296-1301.

17. Nickoloff, B.J. 1991. The cytokine network in psoriasis. Arch. Dermatol. 127:871-884.

18. Zhou, X., et al. 2003. Novel mechanisms of T-cell and dendritic cell activation revealed by profiling of psoriasis on the 63,100-element oligonucleotide array. Physiol. Genomics. 13:69-78.

19. Lomholt, G. 1963. Psoriasis: prevalence, spontaneous course, and genetics; a census study on the prevalence of skin diseases on the Faroe Islands. Copenhagen, Denmark. G.E.C.-Gad. 295 pp.

20. Farber, E.M., and Nall, M.L. 1974. The natural history of psoriasis in 5,600 patients. Dermatologica. 148:1-18.

21. Tiilikainen, A., Lassus, A., Karvonen, J., Vartiainen, P., and Julin, M. 1980. Psoriasis and HLA-Cw6. Br. J. Dermatol. 102:179-184.
22. Gudjonsson, J.E., et al. 2003. Psoriasis patients who are homozygous for the HLA-Cw*0602 allele have a 2.5 -fold increased risk of developing psoriasis compared with Cw6 heterozygotes. Br. J. Dermatol. 148:233-235.

23. Capon, F., Munro, M., Barker, J., and Trembath, R. 2002. Searching for the major histocompatibility complex psoriasis susceptibility gene. J. Invest. Dermatol. 118:745-751.

24. Nair, R.P., et al. 1997. Evidence for two psoriasis susceptibility loci (HLA and $17 \mathrm{q}$ ) and two novel candidate regions ( $16 \mathrm{q}$ and $20 \mathrm{p}$ ) by genome-wide scan. Hum. Mol. Genet. 6:1349-1356.

25. Trembath, R.C., et al. 1997. Identification of a major susceptibility locus on chromosome $6 \mathrm{p}$ and evidence for further disease loci revealed by a two stage genome-wide search in psoriasis. Hum. Mol. Genet. 6:813-820.

26. International Psoriasis Genetics Consortium. 2003. The International Psoriasis Genetics Study: assessing linkage to 14 candidate susceptibility loci in a cohort of 942 affected sib pairs. Am. J. Hum. Genet. 73:430-437.

27. Ishihara, M., et al. 1996. Genetic polymorphisms in the keratin-like S gene within the human major histocompatibility complex and association analysis on the susceptibility to psoriasis vulgaris. Tissue Antigens. 48:182-186.

28. Martin, M.P., et al. 2002. Cutting edge: susceptibility to psoriatic arthritis: influence of activating killer Ig-like receptor genes in the absence of specific HLA-C alleles. J. Immunol. 169:2818-2822.

29. Nickoloff, B.J. 1999. Skin innate immune system in psoriasis: friend or foe? J. Clin. Invest. 104:1161-1164.

30. Tomfohrde, J., et al. 1994. Gene for familial psoriasis susceptibility mapped to the distal end of human chromosome 17q. Science. 264:1141-1145.

31. Speckman, R.A., et al. 2003. Novel immunoglobulin superfamily gene cluster, mapping to a region of human chromosome $17 \mathrm{q} 25$, linked to psoriasis susceptibility. Hum. Genet. 112:34-41.

32. Helms, C., et al. 2003. A putative RUNX1 binding site variant between SLC9A3R1 and NAT9 is associated with susceptibility to psoriasis. Nat. Genet. 35:349-356.

33. Bromley, S.K., et al. 2001. The immunological synapse. Annu. Rev. Immunol. 19:375-396.

34. Lebwohl, M., et al. 2003. A novel targeted T-cell modulator, efalizumab, for plaque psoriasis. N. Engl.J. Med. 349:2004-2013.

35. Nickoloff, B.J., et al. 1993. Activated keratinocytes present bacterial-derived superantigens to T lymphocytes: relevance to psoriasis. J. Dermatol. Sci. 6:127-133.

36. Ellis, C.N., and Krueger, G.G. 2001. Treatment of chronic plaque psoriasis by selective targeting of memory effector T lymphocytes. N. Engl. J. Med. 345:248-255.

37. Abrams, J.R., et al. 2000. Blockade of T lymphocyte costimulation with cytotoxic T lymphocyteassociated antigen 4-immunoglobulin (CTLA4Ig) reverses the cellular pathology of psoriatic plaques, including the activation of keratinocytes, dendritic cells, and endothelial cells. J. Exp. Med. 192:681-694.

38. Weinshenker, B.G., Bass, B.H., Ebers, G.C., and
Rice, G.P. 1989. Remission of psoriatic lesions with muromonab-CD3 (orthoclone OKT3) treatment. J. Am. Acad. Dermatol. 20:1132-1133.

39. Gottlieb, A.B., et al. 2000. Anti-CD4 monoclonal antibody treatment of moderate to severe psoriasis vulgaris: results of a pilot, multicenter, multiple-dose, placebo-controlled study. J. Am. Acad. Dermatol. 43:595-604.

40. Gottlieb, S.L., et al. 1995. Response of psoriasis to a lymphocyte-selective toxin (DAB389IL-2) suggests a primary immune, but not keratinocyte, pathogenic basis. Nat. Med. 1:442-447.

41. Werlen, G., Hausmann, B., Naeher, D., and Palmer, E. 2003. Signaling life and death in the thymus: timing is everything. Science. 299:1859-1863.

42. Sakaguchi, N., et al. 2003. Altered thymic T-cell selection due to a mutation of the ZAP-70 gene causes autoimmune arthritis in mice. Nature. 426:454-460.

43. Alarcon-Riquelme, M.E. 2003. A RUNX trio with a taste for autoimmunity. Nat. Genet. 35:299-300.

44. Prokunina, L., et al. 2002. A regulatory polymorphism in PDCD1 is associated with susceptibility to systemic lupus erythematosus in humans. Nat. Genet. 32:666-669.

45. Tokuhiro, S., et al. 2003. An intronic SNP in a RUNX1 binding site of SLC22A4, encoding an organic cation transporter, is associated with rheumatoid arthritis. Nat. Genet. 35:341-348.

46. Itoh, K., et al. 2002. Cutting edge: negative regulation of immune synapse formation by anchoring lipid raft to cytoskeleton through Cbp-EBP50ERM assembly. J. Immunol. 168:541-544.

47. Rioux, J.D., et al. 2001. Genetic variation in the 5 q31 cytokine gene cluster confers susceptibility to Crohn disease. Nat. Genet. 29:223-228.

48. Yabuuchi, H., et al. 1999. Novel membrane transporter OCTN1 mediates multispecific, bidirection$\mathrm{al}$, and $\mathrm{pH}$-dependent transport of organic cations. J. Pharmacol. Exp. Ther. 289:768-773.

49. Rodriguez, P.C., et al. 2003. L-arginine consumption by macrophages modulates the expression of CD3 zeta chain in T lymphocytes. J. Immunol. 171:1232-1239.

50. Hewett, D., et al. 2002. Identification of a psoriasis susceptibility candidate gene by linkage disequilibrium mapping with a localized single nucleotide polymorphism map. Genomics. 79:305-314.

51. Matzinger, P. 2002. The danger model: a renewed sense of self. Science. 296:301-305.

52. Soumelis, V., et al. 2002. Human epithelial cells trigger dendritic cell mediated allergic inflammation by producing TSLP. Nat. Immunol. 3:673-680.

53. Nickoloff, B.J., and Naidu, Y. 1994. Perturbation of epidermal barrier function correlates with initiation of cytokine cascade in human skin. J. Am. Acad. Dermatol. 30:535-546.

54. Baker, B.S., et al. 2001. Skin T cell proliferative response to $M$ protein and other cell wall and membrane proteins of group A streptococci in chronic plaque psoriasis. Clin. Exp. Immunol. 124:516-521.

55. Travers, J.B., et al. 1999. Epidermal HLA-DR and the enhancement of cutaneous reactivity to superantigenic toxins in psoriasis. J. Clin. Invest. 104:1181-1189.

56. Curry, J.L., Qin, J.Z., Robinson, J., and Nickoloff, B.J. 2003. Reactivity of resident immunocytes 
in normal and prepsoriatic skin using an ex vivo skin-explant model system. Arch. Pathol. Lab. Med. 127:289-296.

57. Bonish, B., et al. 2000. Overexpression of CD1d by keratinocytes in psoriasis and CD1d-dependent IFN-gamma production by NK-T cells. J. Immunol. 165:4076-4085.

58. Gerlini, G., et al. 2001. CD1d is expressed on dermal dendritic cells and monocyte-derived dendritic cells. J. Invest. Dermatol. 117:576-582.

59. Curry, J.L., et al. 2003. Innate immune-related receptors in normal and psoriatic skin. Arch. Pathol. Lab. Med. 127:178-186.

60. Nestle, F.O., Turka, L.A., and Nickoloff, B.J. 1994. Characterization of dermal dendritic cells in psoriasis. Autostimulation of $\mathrm{T}$ lymphocytes and induction of Th1 type cytokines. J. Clin. Invest. 94:202-209.

61. Uyemura, K., Yamamura, M., Fivenson, D.F., Modlin, R.L., and Nickoloff, B.J. 1993. The cytokine network in lesional and lesion-free psoriatic skin is characterized by a T-helper type 1 cell-mediated response. J. Invest. Dermatol. 101:701-705.

62. Schlaak, J.F., et al. 1994. T cells involved in psoriasis vulgaris belong to the Th1 subset. J. Invest. Dermatol. 102:145-149.

63. Reich, K., et al. 2002. Promoter polymorphisms of the genes encoding tumor necrosis factor-alpha and interleukin-1beta are associated with different subtypes of psoriasis characterized by early and late disease onset. J. Invest. Dermatol. 118:155-163.

64. Asadullah, K., et al. 2001. Interleukin-10 promoter polymorphism in psoriasis. J. Invest. Dermatol. 116:975-978.

65. Oestreicher, J.L., et al. 2001. Molecular classification of psoriasis disease-associated genes through pharmacogenomic expression profiling. Pharmacogenomics J. 1:272-287.

66. Bowcock, A.M., et al. 2001. Insights into psoriasis and other inflammatory diseases from largescale gene expression studies. Hum. Mol. Genet. 10:1793-1805.

67. Nathan, C. 2002. Points of control in inflammation. Nature. 420:846-852.

68. Nickoloff, B.J., et al. 1991. Cellular localization of interleukin-8 and its inducer, tumor necrosis factor-alpha in psoriasis. Am. J. Pathol. 138:129-140.

69. Aggarwal, S., Ghilardi, N., Xie, M.H., de Sauvage, F.J., and Gurney, A.L. 2003. Interleukin-23 promotes a distinct CD4 $\mathrm{T}$ cell activation state characterized by the production of interleukin-17. J. Biol. Chem. 278:1910-1914.

70. Lee, E., et al. 2004. Increased expression of interleukin 23 p19 and p40 in lesional skin of patients with psoriasis vulgaris. J. Exp. Med. 199:125-130.

71. Ruckert, R., et al. 2000. Inhibition of keratinocyte apoptosis by IL-15: a new parameter in the pathogenesis of psoriasis? J. Immunol. 165:2240-2250.

72. Villadsen, L.S., et al. 2003. Resolution of psoriasis upon blockade of IL-15 biological activity in a xenograft mouse model. J. Clin. Invest. 112:1571-1580. doi:10.1172/JCI200318986

73. Albanesi, C., et al. 2000. Interleukin-17 is produced by both Th1 and Th2 lymphocytes, and modulates interferon-gamma- and interleukin-4-induced activation of human keratinocytes. J. Invest. Dermatol. 115:81-87.

74. Ohta, Y., Hamada, Y., and Katsuoka, K. 2001. Expression of IL-18 in psoriasis. Arch. Dermatol. Res. 293:334-342.

75. Blumberg, H., et al. 2001. Interleukin 20: discovery, receptor identification, and role in epidermal function. Cell. 104:9-19.

76. Quinones, M.P., et al. 2004. Experimental arthritis in CC chemokine receptor 2-null mice closely mimics severe human rheumatoid arthritis. J. Clin. Invest. 113:856-866. doi:10.1172/JCI200420126.

77. Krueger, G.G., Bergstresser, P.R., Lowe, N.J., Voorhees, J.J., and Weinstein, G.D. 1984. Psoriasis. J. Am. Acad. Dermatol. 11:937-947.

78. Wrone-Smith, T., and Nickoloff, B.J. 1996. Dermal injection of immunocytes induces psoriasis. J. Clin. Invest. 98:1878-1887.

79. Boehncke, W.H., Dressel, D., Zollner, T.M., and Kaufmann, R. 1996. Pulling the trigger on psoriasis. Nature. 379:777.

80. Nickoloff, B.J., and Wrone-Smith, T. 1999. Injection of pre-psoriatic skin with CD4+ T cells induces psoriasis. Am. J. Pathol. 155:145-158.

81. Kess, D., et al. 2003. CD4(+) T cell-associated pathophysiology critically depends on CD18 gene dose effects in a murine model of psoriasis. J. Immunol. 171:5697-5706.

82. Nickoloff, B.J., Bonish, B., Huang, B.B., and Porcelli, S.A. 2000. Characterization of a T cell line bearing natural killer receptors and capable of creating psoriasis in a SCID mouse model system. J. Dermatol. Sci. 24:212-225.

83. Gilhar, A., et al. 2002. Psoriasis is mediated by a cutaneous defect triggered by activated immunocytes: induction of psoriasis by cells with natural killer receptors. J. Invest. Dermatol. 119:384-391.

84. Zeigler, M., et al. 2001. Anti-CD11a ameliorates disease in the human psoriatic skin-SCID mouse transplant model: comparison of antibody to CD11a with Cyclosporin A and clobetasol propionate. Lab. Invest. 81:1253-1261.

85. Schon, M.P., et al. 2002. Efomycine M, a new specific inhibitor of selectin, impairs leukocyte adhesion and alleviates cutaneous inflammation. Nat. Med. 8:366-372.

86. Zollner, T.M., et al. 2002. Proteasome inhibition reduces superantigen-mediated $\mathrm{T}$ cell activation and the severity of psoriasis in a SCID-hu model. J. Clin. Invest. 109:671-679. doi:10.1172/ JCI200212736.

87. Boyman, O., et al. 2004. Spontaneous development of psoriasis in a new animal model shows an essential role for resident $\mathrm{T}$ cells and tumor necrosis factor-\{alpha\}. J. Exp. Med. 199:731-736.

88. Reynolds, N.J., and Al-Daraji, W.I. 2002. Calcineurin inhibitors and sirolimus: mechanisms of action and applications in dermatology. Clin. Exp. Dermatol. 27:555-561.

89. Reitamo, S., et al. 2001. Efficacy of sirolimus (rapamycin) administered concomitantly with a subtherapeutic dose of cyclosporin in the treat- ment of severe psoriasis: a randomized controlled trial. Br. J. Dermatol. 145:438-445.

90. Rappersberger, K., et al. 2002. Pimecrolimus identifies a common genomic anti-inflammatory profile, is clinically highly effective in psoriasis and is well tolerated. J. Invest. Dermatol. 119:876-887.

91. Antoni, C., and Manger, B. 2002. Infliximab for psoriasis and psoriatic arthritis. Clin. Exp. Rheumatol. 20(Suppl. 28):S122-S125.

92. Scheinfeld, N. 2003. Adalimumab (HUMIRA): a review. J. Drugs Dermatol. 2:375-377.

93. Mease, P.J., et al. 2000. Etanercept in the treatment of psoriatic arthritis and psoriasis: a randomized trial. Lancet. 356:385-390.

94. Leonardi, C.L., et al. 2003. Etanercept as monotherapy in patients with psoriasis. N. Engl. J. Med. 349:2014-2022.

95. Rutgeerts, P., et al. 2003. Treatment of active Crohn's disease with onercept (recombinant human soluble p55 tumour necrosis factor receptor): results of a randomized, open-label, pilot study. Aliment. Pharmacol. Ther. 17:185-192.

96. Asadullah, K., et al. 1998. IL-10 is a key cytokine in psoriasis. Proof of principle by IL-10 therapy: a new therapeutic approach. J. Clin. Invest. 101:783-794.

97. Trepicchio, W.L., et al. 1999. Interleukin-11 therapy selectively downregulates type I cytokine proinflammatory pathways in psoriasis lesions. J. Clin. Invest. 104:1527-1537.

98. Ghoreschi, K., et al. 2003. Interleukin-4 therapy of psoriasis induces $\mathrm{Th} 2$ responses and improves human autoimmune disease. Nat. Med. 9:40-46.

99. de Fougerolles, A.R., et al. 2000. Regulation of inflammation by collagen-binding integrins alpha1beta 1 and alpha2beta 1 in models of hypersensitivity and arthritis. J. Clin. Invest. 105:721-729.

100.von Andrian, U.H., and Engelhardt, B. 2003. Alpha4 integrins as therapeutic targets in autoimmune disease. N. Engl. J. Med. 348:68-72.

101. Hugot, J.P., et al. 2001 Association of NOD2 leucine-rich repeat variants with susceptibility to Crohn's disease. Nature. 411:599-603.

102. Ogura, Y., et al. 2001. A frameshift mutation in NOD2 associated with susceptibility to Crohn's disease. Nature. 411:603-606.

103. Rahman, P., et al. 2003. CARD15: a pleiotropic autoimmune gene that confers susceptibility to psoriatic arthritis. Am. J. Hum. Genet. 73:677-681.

104.Lala, S., et al. 2003. Crohn's disease and the NOD2 gene: a role for paneth cells. Gastroenterology. 125:47-57.

105. Weitz-Schmidt, G., et al. 2001. Statins selectively inhibit leukocyte function antigen-1 by binding to a novel regulatory integrin site. Nat. Med. 7:687-692.

106. Kumar, S., Boehm, J., and Lee, J.C. 2003. p38 MAP kinases: key signalling molecules as therapeutic targets for inflammatory diseases. Nat. Rev. Drug Discov. 2:717-726.

107. Changelian, P.S., et al. 2003. Prevention of organ allograft rejection by a specific Janus kinase 3 inhibitor. Science. 302:875-878.

108. Steed, P.M., et al. 2003. Inactivation of TNF signaling by rationally designed dominant-negative TNF variants. Science. 301:1895-1898. 